\title{
An Interactive Table for Regulating Face-to-Face Collaborative Learning
}

\author{
Khaled Bachour, Frédéric Kaplan, and Pierre Dillenbourg \\ Swiss Federal Institute of Technology \\ Lausanne, Switzerland \\ \{khaled.bachour, frederic.kaplan, pierre.dillenbourg\}@epfl.ch \\ No Institute Given
}

\begin{abstract}
In face-to-face collaborative learning, unbalanced participation often leads to the undersirable result of some participants experiencing lower learning outcomes. Providing feedback to the participants on the level of their participation could have a positive effect on the their ability to self-regulate, leading to a more balanced collaboration. We propose a new approach for providing this feedback that takes the shape of a meeting table with a reactive visualization displayed on its surface. The meeting table monitors the collaborative interaction taking place around it using embedded microphones and displays a real-time feedback to the participants on an array of LEDs, inviting them to balance their collaboration. We report on an ongoing study that currently shows a positive effect our table has on group regulation.
\end{abstract}

Keywords: Collaborative Learning, Roomware, Ubiquitous Computing, Interactive Furniture.

\section{Introduction}

In situations of face-to-face collaboration, unbalanced participation often leads to undesirable results. In the context of learning, these results take the form of lower learning outcomes for members of a group that did not participate enough [1,2, 3]. One way to overcome this effect is by encouraging members of a group to participate equally. This could be achieved by indicating to individual members their level of participation in a shared display.

There have been several recent attempts to enhance conversation with visualizations of member participation. Bergstrom and Karahalios [4] present an approach that transforms the conversation history into a interesting graphical representation that they refer to as a Conversation Clock. This "clock" shows individual user contributions in colorcoded bars that run along the perimeter a large circle. As time goes by, the older bars move towards the center of the circle and new bars continue to appear on the outer circle. The result is an interesting snapshot of the conversation that captures the history of the conversation in terms of member participation. This snapshot contains a significant amount of information about the conversation; however, it does not focus the attention of the speakers on one or more specific aspects of that converstion, namely dominance or turn-taking patterns. In a more recent work [5], the authors present another system in which each speaker turn is displayed as a colored bar on a shared display. During 
each speaker turn, other members of the group may anonymously vote for the value of the contribution being made. The resulting display provides a global view of who is speaking more and how the others value each person's contributions.

DiMicco [6, 7] uses a display that is projected on some shared surface such as the tabletop or a wall in order to show relevant information on the conversation taking place. The information displayed varies and can show dominance, turn-taking patterns among other things. DiMicco also explores different settings for the use of such a visualization by varying the detail presented in the visualization as well the the time it is presented (during or after the meeting).

In this paper we present our ongoing work on an interactive table, Reflect, that is similar to the works presented above in that it monitors the conversation taking place around it and displays a visualizaiton of that conversation on its surface. Among these works, Reflect is most similar to DiMicco's approach as we are also interested in highlighting overparticipation and underparticipation. We attempt to offer a visualization pattern that is more focused on participation levels and provides a stronger comparative view of these levels.

In the next section, we motivate the need for balancing participation in group learning situations. We then present the details of our own approach in Section 3 and our ongoing experimental study and preliminary results in Section 4. Some partial results from our ongoing study are presented in Section 5. We describe the possible and planned extensions to the table in Section 6.

\section{Role of Participation}

Most participation in face-to-face meetings takes the form of verbal communication in that members who are silent are seen as not participating or not contributing to the meeting. In the context of learning, this verbalization plays an important role in the formation of concepts and in the students' reassessment of their own understanding of a situation [8].

In this section we discuss how unbalanced participation in group meetings has a tendancy to reduce the effectiveness of the meeting, either in terms of the quality of the decision made, or in terms of the learning outcomes of the participants.

\subsection{Effects on Group Learning}

Whether or not they are required to do so, students often find themselves working together in groups. Empircal research has shown that collaborative learning can be more effective than individual learning [9]. However, this is not always the case.

Cohen [1] describes some criteria for group productivity, without which group learners might benefit less than individual learners. Among these, lack of equity in participation is presented as an obstacle to effective learning in a group. Cohen argues that participation is a predictor of learning gains such that the more individual members participate within a group, the more they learn.

Cohen also suggests that the difference in participation is not necessarily related to participants' abilities or their expertise, but rather it is related to their perceived status 
which can come from any number of stimuli including age, gender, or race of the participant. In some cases, perceived popularity or attractiveness of individuals can lead to more active participation on their part, which in turn leads to lower learning gains for their partners $[1,3]$.

Unbalanced participation in group learning can thus be seen as a deterrant for effective learning. Baker [10] even suggests that without symmetry in participation real collaboration cannot take place. There is thus a need to neutralize the effects of the perceived status on the levels of participation of group members by encouraging all members to participate equally.

\subsection{Information Sharing}

There is often a substantial risk that one or more participants who hold critical information are unable to effectively share this information [2]. Proper information sharing is thus a crucial aspect of effective collaborative work. In reality, however, the variety and number of participants who do in fact contribute in the discussion is often less than is deemed appropriate by post-hoc analysis [11]. As a result, discussions take place with some relevant and potentially critical information missing, leading to suboptimal results. This effect could be mitigated if participants were encouraged to participate in a more balanced manner, permitting all members to contribute.

\section{Reflect: An Interactive Table for Balancing Participation}

In its current form Reflect is a luminous board embedded in a table. It monitors conversations via an array of embedded microphones and shows a visualization of the current state of conversation using a board of color LEDs. Its main objective is two-fold: to support users in balancing their participation while remaining unintrusive to the natural flow of conversations.

At the center of the table, a three-microphone beam-forming array developed by Illusonic [12] permits the system to selectively filter out sounds coming from specific directions. This allows the table to listen to each speaker individually without encumbering the users with head-mounted or tie-clipped microphones.

Beneath the frosted-glass surface of the table, an electronic circuit board with 128 individually-addressable multi-color LEDs provides a low-resolution visual display. This LED board can be programmed to show any desired display. For our initial version of the table, we opted for a column display that clearly shows participation levels of individual speakers.

\subsection{Quantity of Participation vs. Quality of Contribution}

Regulating user participation is achieved by displaying on the surface of the table the different levels of participation of each group member. This takes the form of a column of LEDs that light up as the user speaks. The more each user speaks, the more LEDs in that user's column light up. At first glance, this may not seem a very convincing way of regulating participation, since participation is not solely dependant on speech 


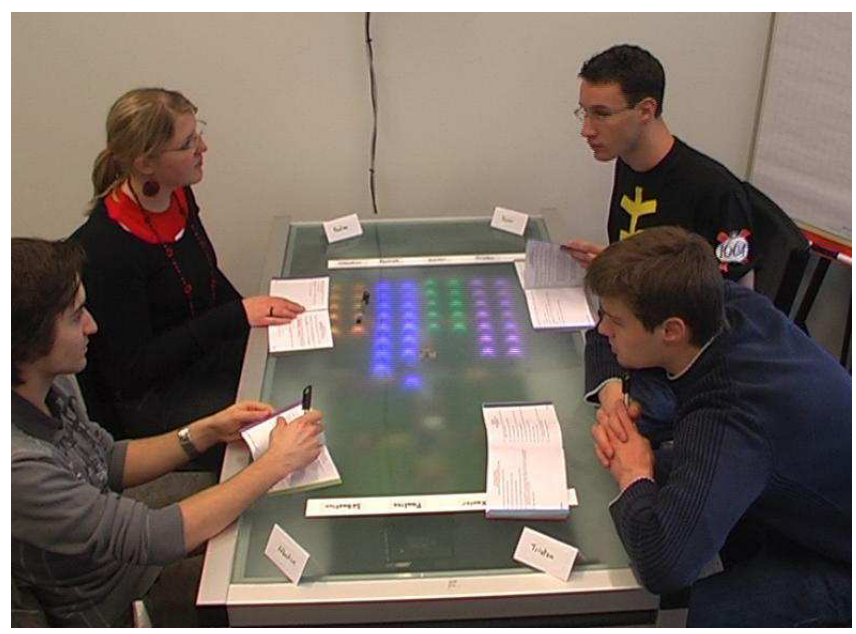

Fig. 1. The current prototype of Reflect.

levels. The member who contributed the most is not necesserily the one who spoke the most. However, at this point, no intelligent system has been conceived for automatically evaluating a person's contribution. We therefore rely on the users' own intelligence in determining whether their low speech level is due to low contribution or due to valuable but brief participation. In any case, we maintain that in the absence of more sophisticated technology, speech levels remain a good though imperfect indicatorx of a user's level of participation. This also allows the table to remain a low-cost and thus more accessible system.

\section{Current Study}

We are currently conducting a study on Reflect in which groups of students are asked to collaboratively solve a task. We present here the details of the study and then report some interesting episodes that show how students responded to the visualisation on the table.

\subsection{Experimental Settings}

Groups of four students are being randomly selected from a pool of bachelor level students that have volunteered for the experiments. Subjects were paid 50 Swiss Francs for their 2-hour involvement in the experiment. Each group is asked to solve a murder mystery task offered to us by Stasser and Stewart [13] and then translated into French to make it more accessible to students in our university community. Each student is given a copy of investigation logs that includes certain important pieces of information that are not available to others. This ensures that all students would be required to participate in the discussion in order to gather all the necessary information. 


\subsection{Experimental Conditions}

There are two experimental conditions that are identical except for the information that is being displayed on the surface of the table. In the first condition, the students are shown their levels of participation i.e. how much time each student talked, and in the second, they are shown the focus of the discussion, i.e. how much time was spent discussing the case of each suspect. The visualizations had the same format for both conditions: a column of LEDs for each student (first condition) or suspect (second condition) would light up in response to the student talking or the suspect being talked about. By simply comparing the length of the columns of lit LEDs, one can easily determine who has spoken most or which suspect was discussed more than the others. A third neutral condition, in which no information is displayed on the table, was not included as it would be quite costly and the benefits of having such a condition are not compelling enough.

\subsection{Experimental Procedure}

The students are first asked to read the investigation logs individually for 30 minutes. They are then allowed to keep the logs with them during the discussion. The table is used to show a simple timer that keeps the students informed of the time remaining.

The students were then given 60 minutes to reach consensus on a given suspect. In order to jumptstart the discussion, the students are asked to come up, for each suspect, with possible means, motive and opportunity for committing the crime. They are informed that to accuse a suspect, they must prove that he has all of these three elements and that the other two suspects are missing one of the elements. The students are made aware that they may possess unique information that is not available to others. Finally the visualizations are explained to the students, but no mention is made of the theoretical benefit of a balanced discussion either in terms of participation or subject discussion.

\section{Partial Results}

The study is still underway, and out of a total of 20 experiments planned ( 80 participants), 8 have already been completed (32 participants). As it is early at this point to attempt to make detailed statistical analysis, we content with some brief quantitative results and focus on some qualitative analysis of some interesting episodes that occured in some of our experiments. In the post experiment questionnaire, we tried to measure the effect of the table in terms of promoting self-regulation among group members. We wanted to answer the following questoin:

- Can a real-time visualization change the way participants behave by promoting self-regulation? 


\subsection{Case Study}

At the end of the study we will attempt to answer this question by statistically analyzing the participants' levels of participation and their ability to estimate these levels. At this point, we focus our discussion to the qualitative aspect of the results. For that we look at the answers to the open questions that were given in the questionnaire. In particular, the questions:

1. Can you indicate one or more occasions where the visual display influenced your behaviour?

2. Can you indicate one or more occations where the visual display had a negative impact on the collaboration?

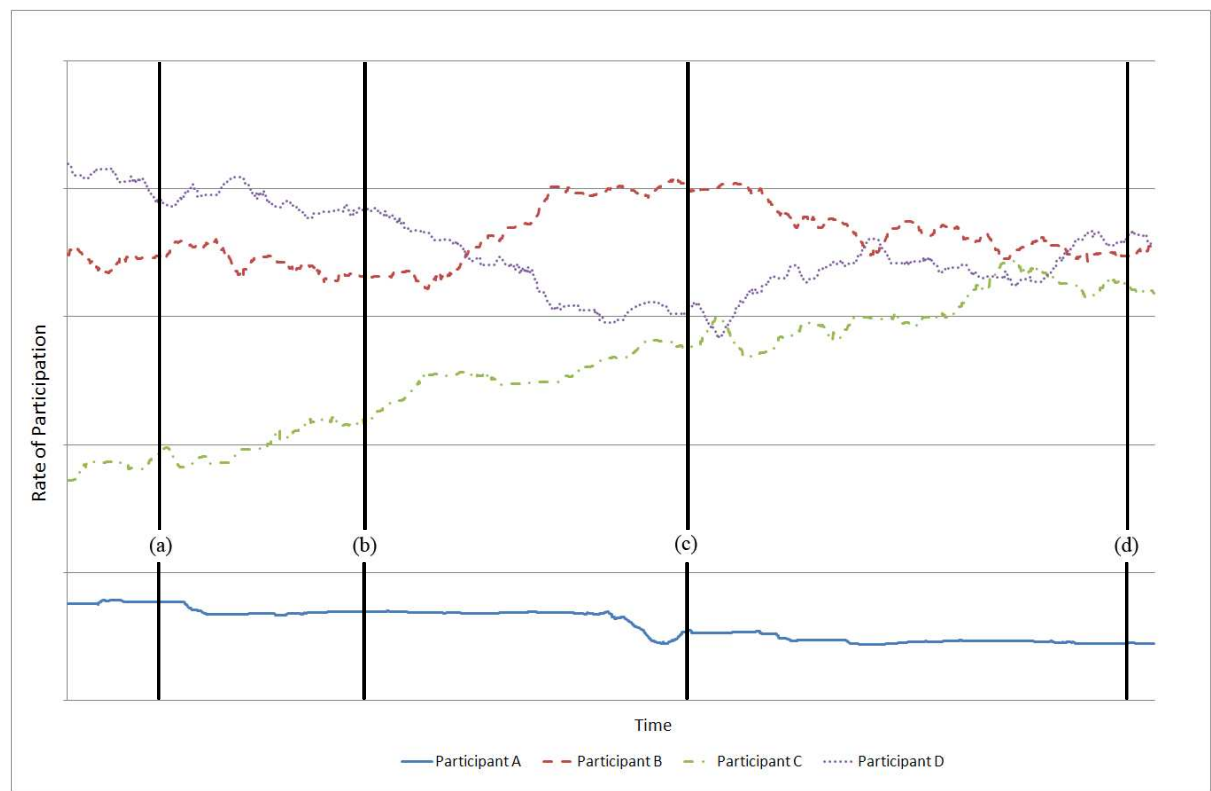

Fig. 2. Rate of participation of members of one group is the amount of speech produced by each member over a certain amount of time. Four points of interests are labeled. The state of the table on these points of interest can be seen in Figure 3

We discuss here a case study of a group who solved the murder mystery task in the first experimental condition. We chose this example because it illustrates both a clear regulatory effect the table had on some members, as well as a clear lack of effect it had on others. Figure 2 shows the rate of participation of each member in this group over time. Some interesting observations can be made about this group discussion.

1. Participant $\mathrm{C}$ responded to the second question by saying that when she noticed that her LEDs weren't lit, she got "frustrated." We can clearly see in the Figure 2 that the 


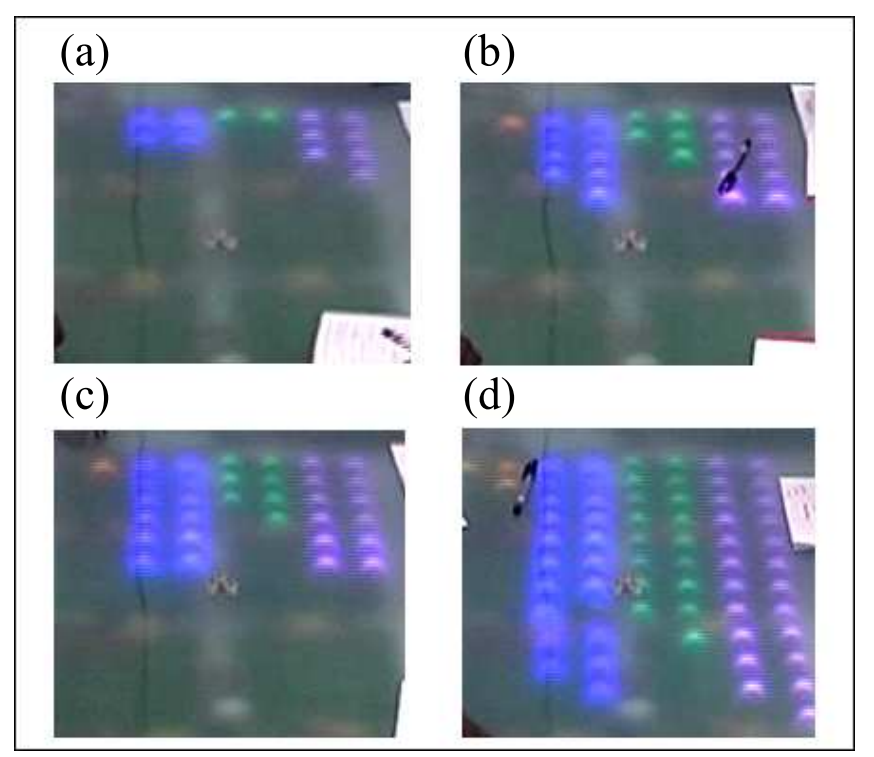

Fig. 3. The state of the table at the four points indicated int Figure 2. 10 minutes into the discussion in (a) the participation is clearly unbalanced. Participant A begins reducing her level of participation. In (b), Participants B and D begin to approach each other while Participant $C$ still lags behind with less than half the total speaking time. In (c), the point in Figure 2 where we see Participant $\mathrm{D}$ begin to increase her participation again, the table shows Participants B and D with equal participation. Participant $\mathrm{C}$ is still increasing her rate of participation at this point. Near the end of the experiment, in (d), Participants B and D have almost equal participation levels, while $\mathrm{C}$ remains slightly behind. Participant A never shows concern for his low participation level.

rate of participation for this student began much lower than that of participants $\mathrm{B}$ and $\mathrm{D}$, but eventually, and for the remainder of the discussion, Participant $\mathrm{C}$ began speaking almost as much as participants B and D.

Although frustration is not a desirable emotion we wish our table to invoke in its users, the end result of self-regulation is beneficial.

2. A clearer example of deliberate self-regulation was observed in Participant $D$ who explicitly noted in her response to the open questions that she "tried not to surpass the speaking time of [Participant B]" and that sometimes she "refrained from talking to avoid having a lot more lights than the others." This is also visible in the graph where we see that Participant D started off participating slightly more than the others. At one point, she reduced her participation level and eventually maintained it at the same rate as Participant B.

3. In contrast, we clearly see the utter lack of effect the table had on participant A who kept his participation at an absolute minimum. This participant said, in response to questions in the questionnaire, that he rarely looked at the table and that he did not feel it is important for members of the group to participate equally. 
Note that the three other participants reported that they looked at the table either sometimes or often, and all three felt that it was important for members of the group to participate equally.

This case study, while far from sufficient, provides insight into the potential regulatory effect this table can have on group discussion. It also highlights some limitations of the table, namely: if a user is not interested in participating in a balanced manner, the table will have little or no effect on their behaviour. In any case, more detailed analysis needs to be made between behaviour of students who use participation level visualizations against those who use a topic-based visualizaiton.

\subsection{Statistical Effect}

We note here an initial test made on the data we currently have. In order to measure the regulatory effect of the table on members' participation levels, we measured the pairwise difference between percentage of participation for members within each group.

We excluded from our results subjects in both conditions who answered "No" in the questionnaire to the question: "Do you think it's important that each participant speak more or less the same amount as others during the discussion?" The reason that these subjects were excluded is that their answer to that question indicates that they were not interested in regulating their participation, and thus their ability to regulate, with or without the help of our visualization, cannot be accuruately measured in this experiment. Moreover, Reflect is not designed as a tool for enforcing regulation, but rather for supporting it. The intention to self-regulate must thus come from the users themselves, and when this intention is missing, any self-regulation the user exhibits would likely be coincidental.

On the remaining pairs of students (26 pairs, 16 in condition with topic-based visualization and 10 in condition with participant-based visualization), we applied a robust test of equality of means, due to a highly significant difference between the variances in each condition. The robust test showed that the table had a positive significant effect on the ability of the group members to regulate their behaviour $(t(18.720)=-3.067, p$ $=0.006$ ). This reasserts the claim we made in our case study indicating that, among members who are interested in regulating their participation, the table seems to have a positive regulatory effect.

\section{Future Work}

The current version of Reflect simply shows levels of participation for each member of the group. We are now considering implementing different patterns to represent some other aspects of conversations, namely turn-taking. We believe it might be interesting to indicate on the table any observable patterns in turn-taking such as when some participant consistently follows another in speech, or a participant consistently interrupting another's speech. Such additional visualizations might further increase the shared awareness of the participants, helping them further self-regulate in ways other than changing their level of participation. 
Additionally, we are interested in extending our table to distinguish different kinds of contributions such as asking questions or giving explanations. We aim to do so by applying machine learning techniques on prosodic features of the speaker's voice in order to not only detect who is contributing, but also how they are contributing.

When asked if the looked at the display on the table, our 32 subjects responded as follows: 15 said "Often", 14 said "Sometimes", 3 said "Rarely" and none said "Never". This is encouraging as it indicates that users are capable of paying attention to the visualization while at the same time conducting their discussion. Knowing this, we can assume that what will be displayed on the table could be of great value to the users. It is for that reason that the focus our future work will also be producing meaningful and easy-to-interpret visualizations to display on the surface of Reflect.

\section{References}

[1] Cohen, E.G.: Restructuring the classroom: conditions for productive small groups. Review of Educational Research 64(1) (Spring 1994) 1-35

[2] DiMicco, J.M., Pandolfo, A., Bender, W.: Influencing group participation with a shared display. In: CSCW '04: Proceedings of the 2004 ACM conference on Computer supported cooperative work, New York, NY, USA, ACM Press (2004) 614-623

[3] M. Webster Jr., J. E. Driskel Jr.: Beauty as status. The American Journal of Sociology 89(1) (July 1983) 140-165

[4] Bergstrom, T., Karahalios, K.: Conversation clock: Visualizing audio patterns in co-located groups. In: HICSS. (2007) 78

[5] Bergstrom, T., Karahalios, K.: Visualizing co-located conversation feedback. In: IEEE TableTop. (2007)

[6] DiMicco, J.M.: Changing Small Group Interaction through Visual Reflections of Social Behavior. PhD thesis, Massachusetts Institute of Technology (2005)

[7] DiMicco, J.M., Bender, W.: Group reactions to visual feedback tools. In: PERSUASIVE. (2007) 132-143

[8] Hoyles, C.: What is the point of group discussion in mathematics? Educational studies in mathematics 16 (1985) 205-214

[9] Slavin, R.E.: Cooperative Learning. Longman, New York (1983)

[10] Baker, M.: Forms of cooperation in dyadic problem-solving. Revue d'intelligence artificielle 16(4-5) (2002) 587-620

[11] Huber, G.P.: A theory of the effects of advanced information technologies on organizational design, intelligence, and decision making. The academy of management review 15(1) (Januray 1990) 47-71

[12] : http://www.illusonic.com.

[13] Stasser, G., Stewart, D.: Discovery of hidden profiles by decision-making groups: Solving a problem versus making a judgment. Journal of Personality and Social Psychology 63(3) (September 1992) 426-434 